\title{
A New Hybrid Protocol for Random Access and Data Transmission Based on Two-Phase ACB Mechanisms for M2M Communications
}

\author{
Yali Wu, ${ }^{1,2}$ Ningbo Zhang, ${ }^{1,2}$ and Guixia Kang ${ }^{1,2}$ \\ ${ }^{1}$ Key Laboratory of Universal Wireless Communications, Beijing University of Posts and Telecommunications, Beijing, China \\ ${ }^{2}$ Science and Technology on Information Transmission and Dissemination in Communication Networks Laboratory, \\ Shijiazhuang, China \\ Correspondence should be addressed to Guixia Kang; gxkang@bupt.edu.cn
}

Received 26 December 2016; Revised 18 March 2017; Accepted 21 March 2017; Published 30 April 2017

Academic Editor: Jose F. Monserrat

Copyright (c) 2017 Yali Wu et al. This is an open access article distributed under the Creative Commons Attribution License, which permits unrestricted use, distribution, and reproduction in any medium, provided the original work is properly cited.

\begin{abstract}
Machine-to-machine (M2M) communication is considered as one of the key enablers for providing of advanced services and applications. Since M2M features a massive number of user equipment (UE) pieces, one of the key issues is the radio access network (RAN) overload problem for massive connections in M2M communications. In order to improve the number of successful accesses (i.e., the M2M UE pieces that successfully transmit data packets) for M2M communications in the current long term evolution (LTE) systems, we propose a new hybrid protocol for random access (RA) and data transmission based on two-phase access class barring (ACB) mechanisms. Furthermore, the joint optimization algorithm of the two-phase ACB factors and the number of resource blocks (RBs) allocated for RA and data transmission is designed to maximize the number of successful accesses. Finally, simulation results demonstrate that our scheme can significantly improve the number of successful accesses and achieve performance improvement in reducing the grant time.
\end{abstract}

\section{Introduction}

As a key component of Internet of Things (IoT), machine-tomachine (M2M) communications, also known as machinetype communications (MTC) in the third-generation partnership project (3GPP), is being regarded as one of the promising technologies in future 5th-generation $(5 \mathrm{G})$ wireless communications [1]. Recent advances and developments in standards, protocols, and architecture for MTC evolution from 4 th-generation $(4 \mathrm{G})$ to $5 \mathrm{G}$ wireless communications are discussed in [2]. Due to the diverse set of MTC applications and services [3], the current view on the 5G wireless system categorizes MTC into two: (1) massive MTC (mMTC) supplying a massive number of low-data rate and low-cost devices and (2) ultra-reliable low-latency MTC (uMTC) supporting message transmission with high reliability and low latency [4]. An estimated 25 billion pieces of M2M user equipment (UE) will be deployed in 2020 [5]. However, the resulting connection requests from a massive amount of
M2M UE will overwhelm the radio access network (RAN) and degrade the performance of existing human-to-human $(\mathrm{H} 2 \mathrm{H})$ communications [6]. In 3GPP Releases 12 and 13, enhanced MTC (eMTC) is introduced to provide improvements in terms of overload control, signaling overhead reduction, device cost reduction, coverage enhancement, and power saving for longer battery life [7-9]. In 3GPP Release 13 , a narrowband system has been proposed to support M2M communications, which can realize low-cost, low-power, and wide-area cellular connectivity [10]. Further enhancements of narrowband system carry on in Release 14, which is still in progress [11]. A contention-based random access (RA) strategy with direct data transmission is discussed in [4]. This strategy, often termed "direct RA," exploits novel mediumaccess control schemes as well as novel physical layer algorithms to efficiently alleviate the number of connected devices and therefore reduce uplink data traffic congestion.

In this paper, the issues related to the deployment of mMTC using 3GPP long term evolution (LTE) network 
infrastructure is mainly considered. There have been several studies on the RAN overload control and access success probability improvement. Access class barring (ACB) mechanism is proposed in the $3 \mathrm{GPP}$ standard for congestion control. The key of ACB mechanism is to let the eNB broadcast the ACB factor. Each active M2M UE generates a random number between 0 and 1 . If the random number is less than the ACB factor, we say that the active UE can conduct RA procedure. Otherwise, the UE will be randomly barred for a backoff time. In LTE systems, an M2M UE transmits a preamble on the physical random access channel (PRACH) in the first step of RA procedure to inform the evolved nodeB (eNB) of its connection request [12]. In [13], the authors propose a dynamic PRACH resource allocation for clustered M2M UE. The available preambles are dynamically partitioned for each cluster, and the UE belonging to different clusters adopts the ACB mechanism to alleviate the overload. However, the ACB factors are determined through a mass of simulations. In [14-16], the authors propose a traffic-aware ACB strategy to increase the access success probability, where the ACB factor dynamically changes with the varying number of active M2M UE pieces. However, the estimation scheme on the number of active M2M UE pieces has relatively high complexity. In [17], a joint optimal PRACH resource allocation and ACB scheme is proposed to increase access success probability. However, the maximum number of random access opportunities (i.e., the product of preambles number and the PRACH subframes number) is fixed, and it may not be the optimal number when the number of active M2M UE pieces exceeds the maximum number of random access opportunities, which results in the inefficient using of radio resource. In [13-17], the emphasis on the amount of resources for PRACH may not be sufficient since the uplink available resources are limited. The more the PRACH resources allocated to improve the performance of RA procedure, the less the radio resources available for uplink data transmission. In [18], a joint adaptive resource allocation and ACB scheme is proposed to achieve RAN overload control and data transmission at the same time. Both the congestion in PRACH and data channels are emphasized. However, if there is more than one M2M UE piece select the same preamble, and the eNB schedules the same physical uplink shared channel (PUSCH) to this M2M UE for data packet transmissions; this PUSCH is wasted because the eNB cannot decode any data packet. The PUSCH usage efficiency drops greatly due to the serious preamble collisions.

In this paper, the successful access is defined as the M2M UE that successfully transmits its data packet. To improve the number of successful accesses as well as the resource efficiency, we propose a new hybrid RA protocol for RA and data transmissions based on two-phase ACB mechanisms for M2M communications, which increases the number of successful accesses by more efficiently using the radio resources and ACB mechanisms. The work of this paper is summarized as follows.

(1) We extend the current $A C B$ technique that is in the current LTE standard to two-phase ACB mechanisms. In the first phase, the number of participating M2M UE pieces is controlled by the first ACB mechanism.
In the second phase, the participating M2M UE in the first phase is partitioned into two parts by the second ACB mechanism, which, respectively, selects different type of preamble, that is, the nonspecified preamble or the specified preamble.

(2) The nonspecified preamble, which is selected by only one piece of M2M UE, is regarded as successful preamble in this paper. To improve the number of successful preamble, we adaptively adjust the ACB factor in the second phase according to the ratio of nonspecified preambles number and participating M2M UE pieces number. Besides, the number of nonspecified preambles is determined by the number of resource blocks (RBs) allocated to PRACH.

(3) The eNB schedules data channels only for the participating M2M UE that selects the successful preambles and the specified preamble. To improve the number of successful accesses, we realize optimal RBs allocation between PRACH and PUSCH such that all the participating M2M UE pieces that select the successful preambles and the specified preamble can efficiently use PUSCH.

(4) The analytic model is validated by simulation results. We demonstrate that the proposed protocol can significantly improve the number of successful accesses and achieve performance improvement in reducing grant time.

The remainder of this paper is organized as follows. Section 2 introduces the traditional RA procedure in LTE systems. Section 3 presents the system model. Section 4 discusses the details of overload control and resource allocation for the proposed hybrid protocol. Performance evaluations are given in Section 5, while the paper is concluded in Section 6.

\section{Traditional Random Access Procedure in LTE Systems}

The RA procedure in conventional 3GPP LTE systems is classified into contention-based and contention-free [12]. This paper only focuses on contention-based manner. The contention-based RA procedure is shown in Figure 1, which includes the following four steps [12].

Step 1. Preamble transmission: An M2M UE randomly selects a preamble from all available preambles with equal probability and transmits it on PRACH.

Step 2. Random access response (RAR): After detecting the preamble, eNB transmits the corresponding RAR through downlink channels. The RAR conveys the identity of detected preamble, uplink resource grant for the transmission of connection setup request in Step 3, timing alignment (TA) information, and the assignment of a temporary identifier.

Step 3. Connection setup request: After receiving the corresponding RAR, UE adjusts uplink transmission time according to the received TA and transmits the connection setup 


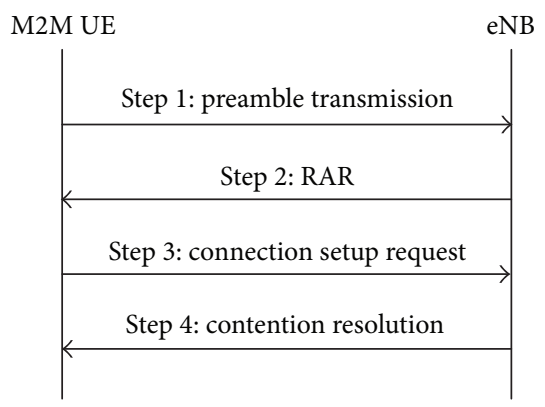

FIGURE 1: The contention-based RA procedure in LTE systems.

request by using the uplink resource grant indicated by the RAR.

Step 4. Contention resolution: If the eNB succeeds in receiving the connection setup request in Step 3, it sends the contention resolution to the corresponding UE. The RA procedure is completed if the UE receives the contention resolution message.

After the UE succeeds in the RA procedure, the UE moves to the connected mode and goes through additional higher layer signaling procedures before it can send data [18]. Since the M2M UE pieces ending up their data transmissions will disconnect from the eNB immediately, the excessive signaling overhead is induced in the case of transmitting small-sized data in conventional RA procedure [7]. Therefore, the RA procedure in conventional 3GPP LTE systems is not suitable for mMTC applications due to the overhead of signaling [20-22]. A contention-based RA strategy with direct data transmission is discussed in $[4,18]$ to reduce the signaling overhead. One-stage access protocol is considered as a low signaling overhead alternative for the RA with mMTC services [23]. These types of RA protocols are also put forward in $[24,25]$. Consequently, in this paper, the data communication procedure is simplified by allowing M2M UE to send data right after preamble transmission, which avoids explicitly establishing a connection.

Furthermore, in conventional RA procedure, if more than one UE piece selects the same preamble in Step 1, they will receive the same RAR and send their connection setup requests on the same PUSCH. In this case, the eNB cannot decode either one due to the cochannel interference; therefore, this PUSCH is wasted.

\section{System Model}

We define the M2M UE attempting to access the network as active M2M UE. We consider a scenario that $N$ active M2M UE pieces exist in the coverage area of an eNB. Let $T_{P}$ represent the activation time, and each M2M UE piece is activated at time $t \in\left[0, T_{P}\right]$ according to a beta probability distribution function $f(t)$ [26].

$$
f(t)=\frac{t^{\alpha-1}\left(T_{P}-t\right)^{\beta-1}}{T_{P}{ }^{\alpha+\beta-1} B(\alpha, \beta)},
$$

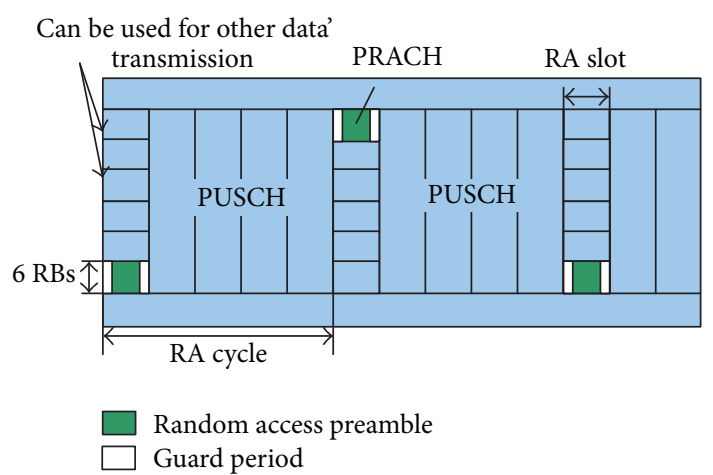

Figure 2: Periodic time-frequency resources for the proposed protocol.

where $B(\alpha, \beta)=\int_{0}^{1} t^{\alpha-1}(1-t)^{\beta-1} d t$. Let $\alpha=3, \beta=4$ [27]; then $f(t)$ is given by

$$
f(t)=\frac{t^{2}\left(T_{P}-t\right)^{3}}{T_{P}^{6} \int_{0}^{1} t^{2}(1-t)^{3} d t}=\frac{60 t^{2}\left(T_{P}-t\right)^{3}}{T_{P}^{6}} .
$$

$T_{P}$ is divided into $I_{P}$ cycles. The $i$ th cycle begins at $t_{i-1}$ and ends at $t_{i}, i=1,2, \ldots, I_{P}$. The first cycle starts from $t_{0}=0$. The last one ends at $t_{I_{\mathrm{P}}}=T_{P}$. According to [26], the expected number of new active M2M UE pieces during the $i$ th cycle is

$$
\lambda_{i}=N \int_{t_{i-1}}^{t_{i}} f(t) d t
$$

To simplify the model, we assume that the new active M2M UE pieces within the $i$ th cycle, that is, within $\left[t_{i-1}, t_{i}\right]$, will only take place at the beginning of this cycle and choose this cycle for their first RA attempts. Apart from these new active M2M UE pieces, reattempting M2M UE pieces arrive at the system for their RA attempts again. The number of reattempting $\mathrm{UE}$ pieces in the $i$ th cycle is $U_{i-1}-V_{i-1}^{\text {succ }}$, where $U_{i-1}$ and $V_{i-1}^{\text {succ }}$ denote the total number of active M2M UE pieces and the number of active M2M UE pieces that successfully transmit data packets in the $(i-1)$ th cycle, respectively. It is assumed that the M2M UE pieces ending up their transmissions in the $(i-1)$ th cycle will disconnect from the eNB immediately, and the reattempting M2M UE pieces in the $(i-1)$ th cycle will reattempt RA procedure in the $i$ th cycle. Therefore, by adding up the number of the reattempting M2M UE pieces that failed in previous cycle and the new active M2M UE pieces in current cycle, we can calculate the total number of active M2M UE pieces in the $i$ th cycle as

$$
U_{i}=U_{i-1}-V_{i-1}^{\text {succ }}+\lambda_{i}
$$

It is known that a UE starts a RA procedure by sending a preamble to the eNB. The PRACH is formed by periodic time-frequency resources for preamble transmission [12]. As shown in Figure 2, a PRACH is regarded as a RA slot. In time domain, the duration of RA slot depends on the preamble format. In frequency domain, a PRACH consists of 6 physical RBs in a subframe, which occupies 864 subcarriers, assuming 
the preamble sequences are mapped to the central 839 subcarriers while the remaining 25 subcarriers are reserved for guard band. To improve the resource efficiency, we extend the traditional preamble transmission by transmitting not only preamble but also the UE identity (UE ID) information and a cyclic redundancy check (CRC) on PRACH $[19,28]$. All the UE ID and CRC information are mapped to the same position in the guard band. Code division multiple access (CDMA) is used to distinguish each UE piece, namely, the UE ID and CRC bits are encoded by a gold sequence. Every preamble has a unique gold sequence; thus when more than one UE piece selects the same preamble, their UE ID will be multiplied by the same sequence. When more than one UE piece transmits their IDs on the same subcarriers, the eNB cannot decode UE ID. Then the eNB thinks collision occurs and will not schedule PUSCH to the preamble, thus saving resource.

Let $S_{i}$ represent the number of RBs allocated to PRACH in the $i$ th cycle, and $S_{i}$ is integer multiples of 6 . Assuming $\kappa$ preamble sequences are mapped to one PRACH, $\kappa S_{i} / 6$ preambles can be constructed in the $i$ th cycle. The eNB chooses one of the constructed preambles as a specified preamble; then the remaining $\kappa S_{i} / 6-1$ preambles are the nonspecified preambles. Denote $Q$ as the total number of RBs allocated for PRACH and PUSCH in a RA cycle and $\varsigma$ as the number of RBs constituting one PUSCH. Then $\left\lfloor\left(Q-S_{i}\right) / \varsigma\right\rfloor$ data channels can be constructed; \lfloor\rfloor denotes the bottom integer function, assuming that an M2M UE uses only one data channel for a fix-sized data packet transmission.

\section{Overload Control and Resource Allocation for the Hybrid Protocol}

\subsection{Proposed Hybrid Protocol for RA and Data Transmission. The proposed hybrid protocol for RA and data transmission consists of six steps as follows.}

Step 1. Before the $i$ th cycle begins, the eNB estimates the number of active M2M UE pieces (i.e., $\widehat{U}_{i}$ ) based on the observation obtained by the $(i-1)$ th cycle. The load estimation algorithm will be explained in Section 4.2. Then the eNB decides the first ACB factor (i.e., $R_{i}^{+}$), the second ACB factor (i.e., $R_{i}^{*}$ ), and the number of RBs for PRACH (i.e., $S_{i}^{*}$ ) from this estimation. As discussed in Section $4.3, R_{i}^{+}=$ $\min \left\{\lfloor Q / \varsigma\rfloor / \widehat{U_{i}}, 1\right\}$. That means if $\widehat{U}_{i}>\lfloor Q / \varsigma\rfloor, R_{i}^{+}=\lfloor Q / \varsigma\rfloor / \widehat{U_{i}}$; otherwise, $R^{+}=1$. After $R^{+}$is determined, $R_{i}^{*}$ is obtained correspondingly. Note that $R_{i}^{*}=\left(\kappa S_{i}^{*} / 6-1\right) / \widehat{U}_{i} R_{i}^{+}$, where $S_{i}^{*}$ is also given in Section 4.3. Then the eNB broadcasts $R_{i}^{+}, R_{i}^{*}$, and $S_{i}^{*}$ to all M2M UE pieces.

Step 2. Each active M2M UE piece randomly selects $x$ out of the uniform distribution between zero and one. If $x \leq R_{i}^{+}$, the active M2M UE can participate in RA procedure. The participating M2M UE randomly selects $y$ out of the uniform distribution between zero and one. If $x>R_{i}^{+}$, the active M2M $\mathrm{UE}$ reattempts RA procedure in the next cycle.

Step 3. Preamble transmission: If $y \leq R_{i}^{*}$, the participating M2M UE randomly selects one preamble out of $\kappa S_{i}^{*} / 6-1$ nonspecified preambles with equal probability. The probability that a nonspecified preamble is selected by the participating M2M UE is $R_{i}^{*} /\left(\kappa S_{i}^{*} / 6-1\right)$. The participating M2M UE transmits the preamble, UE ID information, and CRC on PRACH. If $y>R_{i}^{*}$, the participating M2M UE selects the specified preamble and only transmits the specified preamble on PRACH. The expected number of the participating M2M UE pieces which select the specified preamble is $\widehat{U}_{i} R_{i}^{+}(1-$ $\left.R_{i}^{*}\right)=\widehat{U}_{i} R_{i}^{+}-\left(\kappa S_{i}^{*} / 6-1\right)$.

Step 4. After detecting a preamble, the eNB begins to decode the UE ID. After successfully decoding the ID, the eNB sends the corresponding RAR through downlink channels. The RAR conveys the identity of detected preambles, uplink resource grant for data packet transmission, and TA information. If the eNB cannot decode the UE ID, this preamble is regarded as a collided preamble or the specified preamble. If this preamble is not the specified one, the eNB will stop sending RAR; that is, the eNB will not schedule PUSCH to the collided preamble, thus saving resource, while if it is the specified preamble, the eNB determines how many unscheduled PUSCH are available and considers theses data channels for M2M UE which had selected the specified preamble. Let the number of unscheduled PUSCH be $\widehat{U_{i}} R_{\dot{1}}^{+}-\left(\kappa S_{i}^{*} / 6-1\right)$; that is, the eNB schedules the equal expected number of data channels to the expected number of participating M2M UE pieces which select the specified preamble. The eNB assigns $\widehat{U_{i}} R_{i}^{+}-\left(\kappa S_{i}^{*} / 6-1\right)$ RARs to the specified preamble and sends them in the downlink. The preamble information contained in these RARs is the specified preamble, while the PUSCH information contained in each RAR is different.

Step 5. After receiving the corresponding RAR, the M2M UE adjusts uplink transmission time according to the received TA and transmits a data packet to the eNB on the PUSCH indicated by the RAR.

Step 6. If the eNB correctly detects the data packet transmitted in Step 5, it sends an acknowledgement (ACK) message to the corresponding M2M UE. If an M2M UE receives this message, the M2M UE is regarded as a successful access. If an M2M UE cannot receive this message, it reattempts RA procedure in the next cycle. The proposed hybrid protocol for RA and data transmission procedure is illustrated in Figure 3.

4.2. Load Estimation Algorithm. In this section, a load estimation algorithm is designed to estimate the number of active M2M UE pieces in each cycle. As shown in Figure 4, each preamble can be categorized into the following cases.

(i) Idle preamble, which is not selected by any M2M UE;

(ii) specified preamble;

(iii) successful preamble, that is, nonspecified preamble which is selected by only one M2M UE;

(iv) collided preamble, that is, nonspecified preamble which is selected by more than one M2M UE piece.

As the proposed hybrid protocol shows, the eNB schedules data channels only for the participating M2M UE 


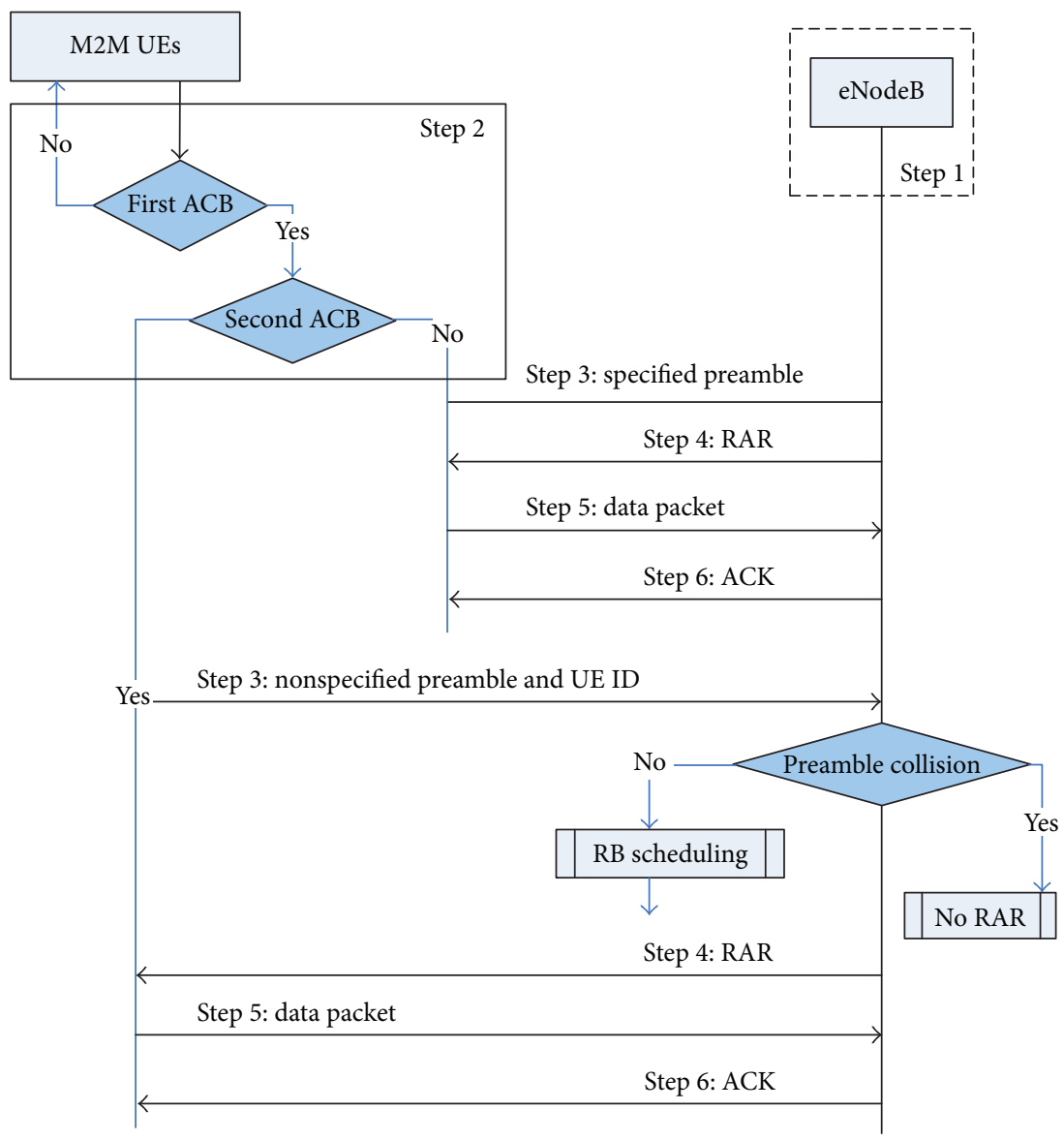

FIgURE 3: The proposed hybrid protocol for RA and data transmission.

pieces that select the successful preambles and the specified preamble. Let $V_{i-1}^{\text {succ, } 1}$ and $V_{i-1}^{\text {succ, } 2}$ represent the number of successful accesses from selecting the successful preambles and the specified preamble in the $(i-1)$ th cycle, respectively. Then, the total number of successful accesses in the $(i-1)$ th cycle is given by $V_{i-1}^{\text {succ }}=V_{i-1}^{\text {succ, } 1}+V_{i-1}^{\text {succ, } 2}$.

Let $V_{i-1}^{\text {coll }}$ represent the number of collided preambles in the $(i-1)$ th cycle. The estimated expected number of active M2M UE pieces in the $i$ th cycle is given by

$$
\begin{aligned}
\widehat{U}_{i}= & \mathrm{E}\left[\lambda_{i}+U_{i-1}-V_{i-1}^{\mathrm{succ}} \mid V_{i-1}^{\mathrm{coll}}\right] \\
= & \lambda_{i}+\mathrm{E}\left[U_{i-1}\left(1-R_{i-1}^{+}\right) \mid V_{i-1}^{\mathrm{coll}}\right] \\
& +\mathrm{E}\left[U_{i-1} R_{i-1}^{+} R_{i-1}^{*}-V_{i-1}^{\mathrm{succ}, 1} \mid V_{i-1}^{\mathrm{coll}}\right] \\
& +\mathrm{E}\left[U_{i-1} R_{i-1}^{+}\left(1-R_{i-1}^{*}\right)-V_{i-1}^{\text {succ }, 2} \mid V_{i-1}^{\mathrm{coll}}\right] .
\end{aligned}
$$

The expected number of active M2M UE pieces failing to participate in RA in the $(i-1)$ th cycle is irrelative with the collided preambles, which is given by

$$
\mathrm{E}\left[U_{i-1}\left(1-R_{i-1}^{+}\right) \mid V_{i-1}^{\mathrm{coll}}\right]=\widehat{U_{i-1}}\left(1-R_{i-1}^{+}\right) .
$$

Since the eNB schedules enough data channels for the participating M2M UE pieces which select the specified

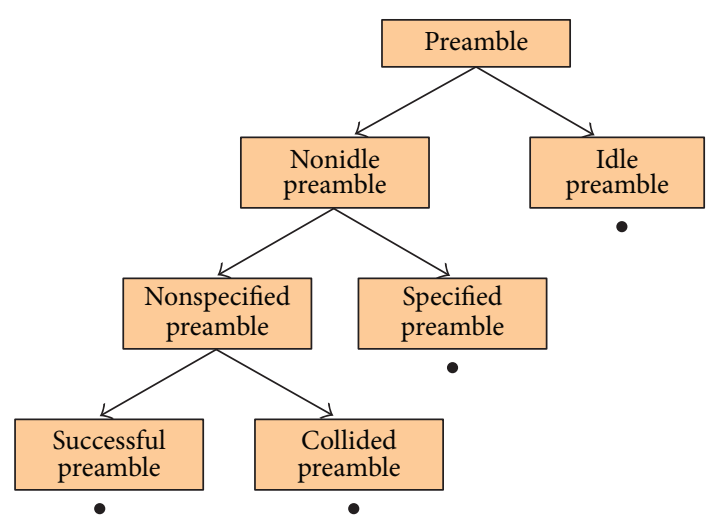

FIgURE 4: The categorization of preamble.

preamble, then all the expected number of participating M2M UE pieces selecting the specified preamble can successfully transmit data packets. We can get

$$
\begin{aligned}
\mathrm{E} & {\left[U_{i-1} R_{i-1}^{+}\left(1-R_{i-1}^{*}\right)-V_{i-1}^{\mathrm{succ}, 2} \mid V_{i-1}^{\mathrm{coll}}\right] } \\
& =\mathrm{E}\left[U_{i-1} R_{i-1}^{+}\left(1-R_{i-1}^{*}\right)\right]-\mathrm{E}\left[V_{i-1}^{\mathrm{succ}, 2}\right]=0,
\end{aligned}
$$


where

$$
\mathrm{E}\left[U_{i-1} R_{i-1}^{+}\left(1-R_{i-1}^{*}\right)\right]=\widehat{U_{i-1}} R_{i-1}^{+}\left(1-R_{i-1}^{*}\right) .
$$

We denote $M_{j \in B_{i-1}}$ as the number of participating M2M UE pieces selecting an nonspecified preamble, named $j$, from $B_{i-1}$ nonspecified preambles in the $(i-1)$ th cycle. The expected number of participating M2M UE pieces selecting the collided preambles in the $(i-1)$ th cycle is given by

$$
\begin{aligned}
& \mathrm{E}\left[U_{i-1} R_{i-1}^{+} R_{i-1}^{*}-V_{i-1}^{\text {succ, } 1} \mid V_{i-1}^{\text {coll }}\right] \\
& =\mathrm{E}\left[M_{j \in B_{i-1}} \mid M_{j \in B_{i-1}} \geq 2\right] V_{i-1}^{\text {coll }} .
\end{aligned}
$$

Under the condition that preamble $j$ is in collision, the expected number of M2M UE pieces that select preambles $j$ is

$$
\begin{aligned}
& \mathrm{E}\left[M_{j \in B_{i-1}} \mid M_{j \in B_{i-1}} \geq 2\right] \\
& =\sum_{k=2}^{\widehat{U_{i-1}} R_{i-1}^{+}} k \cdot \operatorname{Pr}\left\{M_{j \in B_{i-1}}=k \mid M_{j \in B_{i-1}} \geq 2\right\} \\
& =\frac{\sum_{k=1}^{\widehat{U_{i-1}} R_{i-1}^{+}} k \operatorname{Pr}\left\{M_{j \in B_{i-1}}=k\right\}-\operatorname{Pr}\left\{M_{j \in B_{i-1}}=1\right\}}{\operatorname{Pr}\left\{M_{j \in B_{i-1}} \geq 2\right\}} \\
& =\frac{\widehat{U_{i-1}} R_{i-1}^{+} p_{i-1}-\widehat{U_{i-1}} R_{i-1}^{+} p_{i-1}\left(1-p_{i-1}\right)^{\widehat{U_{i-1}} R_{i-1}^{+}-1}}{1-\left(1-p_{i-1}\right)^{\widehat{U_{i-1}} R_{i-1}^{+}}-\widehat{U_{i-1}} R_{i-1}^{+} p_{i-1}\left(1-p_{i-1}\right)^{\widehat{U_{i-1}} R_{i-1}^{+}-1}},
\end{aligned}
$$

where $\operatorname{Pr}\left\{M_{j \in B_{i-1}}=k\right\}$ is the probability that the number of participating M2M UE pieces selecting a nonspecified preamble, named $j$, from $B_{i-1}$ nonspecified preambles in the $(i-1)$ th cycle is $k . \operatorname{Pr}\left\{M_{j \in B_{i-1}}=k\right\}$ is given by

$$
\begin{aligned}
\operatorname{Pr} & \left\{M_{j \in B_{i-1}}=k\right\} \\
& =\operatorname{binom}\left(\widehat{U_{i-1}} R_{i-1}^{+}, k\right) p_{i-1}^{k}\left(1-p_{i-1}\right)^{\widehat{U_{i-1}} R_{i-1}^{+}-k},
\end{aligned}
$$

where $\operatorname{binom}(x, y)=x ! /((y !) \times(x-y) !) \cdot p_{i-1}$ is the probability that a nonspecified preamble is selected by M2M UE, and $p_{i-1}=R_{i-1}^{*} /\left(\kappa S_{i-1}^{*} / 6-1\right)$. Since $R_{i-1}^{*}=\left(\kappa S_{i-1}^{*} / 6-1\right) / \widehat{U_{i-1}} R_{i-1}^{+}$, then $p_{i-1}=1 / \widehat{U_{i-1}} R_{i-1}^{+}$. Therefore, (10) is

$$
\begin{aligned}
& \mathrm{E}\left[M_{j \in B_{i-1}} \mid M_{j \in B_{i-1}} \geq 2\right] \\
& =\frac{1-\left(1-1 /\left(\widehat{U_{i-1}} R_{i-1}^{+}\right)\right)^{\widehat{U_{i-1}} R_{i-1}^{+}-1}}{1-\left(1-1 /\left(\widehat{U_{i-1}} R_{i-1}^{+}\right)\right)^{\widehat{U_{i-1}} R_{i-1}^{+}-1}\left(2-1 /\left(\widehat{U_{i-1}} R_{i-1}^{+}\right)\right)} .
\end{aligned}
$$

From (5)-(7), (9), and (12), we can calculate

$$
\begin{aligned}
\widehat{U_{i}} & =\lambda_{i}+\widehat{U_{i-1}}\left(1-R_{i-1}^{+}\right) \\
& +\frac{1-\left(1-1 /\left(\widehat{U_{i-1}} R_{i-1}^{+}\right)\right)^{\widehat{U_{i-1}} R_{i-1}^{+}-1}}{1-\left(1-1 /\left(\widehat{U_{i-1}} R_{i-1}^{+}\right)\right)^{\widehat{U_{i-1}} R_{i-1}^{+}-1}\left(2-1 /\left(\widehat{U_{i-1}} R_{i-1}^{+}\right)\right)} \\
& \cdot V_{i-1}^{\text {coll }} .
\end{aligned}
$$

After the $(i-1)$ th cycle, the eNB obtains the knowledge of $R_{i-1}^{+}$and $V_{i-1}^{\text {coll }}$ and then updates $\widehat{U_{i-1}}$ to $\widehat{U}_{i}$, based on (13).
4.3. Joint Optimization of the ACB Factors and the Number of $R B$ s for $R A$ and Data Transmission. As previously mentioned, $\left\lfloor\left(Q-S_{i}\right) / \varsigma\right\rfloor$ data channels can be constructed; then the maximum number of participating M2M UE pieces that can be supported by the data channels is $\lfloor Q / \varsigma\rfloor$ if $S_{i}=0$. The number of participating M2M UE pieces is controlled to be no more than $\lfloor Q / \varsigma\rfloor$; then the first $\mathrm{ACB}$ factor is determined by $R_{i}^{+}=\min \left\{\lfloor Q / \varsigma\rfloor / \widehat{U_{i}}, 1\right\}$. Suppose the second ACB factor is $R_{i}$. The expected number of participating M2M UE pieces that select the successful preambles and the specified preamble is

$$
C_{i}=\mathrm{E}\left[V_{i}^{\mathrm{one}}\right]+\widehat{U}_{i} R_{i}^{+}\left(1-R_{i}\right),
$$

where $\mathrm{E}\left[V_{i}^{\text {one }}\right]$ is the expected number of participating $\mathrm{M} 2 \mathrm{M}$ UE pieces that select the successful preambles.

Theorem 1. $\mathrm{E}\left[V_{i}^{\text {one }}\right]$ is given by

$$
\mathrm{E}\left[V_{i}^{\text {one }}\right]=\widehat{U}_{i} R_{i}^{+} R_{i}\left(1-\frac{R_{i}}{\kappa S_{i} / 6-1}\right)^{\widehat{U_{i}} R_{i}^{+}-1} .
$$

The second $A C B$ factor to maximize $\mathrm{E}\left[V_{i}^{\text {one }}\right]$ is given by

$$
R_{i}=\min \left(1, \frac{\kappa S_{i} / 6-1}{\widehat{U}_{i} R_{i}^{+}}\right) .
$$

Proof. See Appendix.

If $R_{i}=1$, all the participating M2M UE pieces randomly select an nonspecified preamble out of $\kappa S_{i} / 6-1$ nonspecified preambles. Since the participating M2M UE pieces are partitioned into two parts for selecting nonspecified preambles and specified preamble, we let $R_{i}=\left(\kappa S_{i} / 6-1\right) / \widehat{U_{i}} R_{i}^{+}$, that is, $R_{i}<1$. Let $\max \left(\mathrm{E}\left[V_{i}^{\text {one }}\right]\right)$ denote the value of $\mathrm{E}\left[V_{i}^{\text {one }}\right]$ when $R_{i}=\left(\kappa S_{i} / 6-1\right) / \widehat{U}_{i} R_{i}^{+}$, and then

$$
\max \left(\mathrm{E}\left[V_{i}^{\text {one }}\right]\right)=\left(\frac{\kappa S_{i}}{6}-1\right)\left(1-\frac{1}{\widehat{U_{i} R_{i}^{+}}}\right)^{\widehat{U_{i} R_{i}^{+}-1}} .
$$

Also, $\widehat{U}_{i} R_{i}^{+}\left(1-R_{i}\right)=\widehat{U}_{i} R_{i}^{+}-\left(\kappa S_{i} / 6-1\right)$ when $R_{i}=\left(\kappa S_{i} / 6-\right.$ 1) $\widehat{U_{i}} R_{i}^{+}$. Then, we have

$$
\begin{aligned}
C_{i}= & \max \left(\mathrm{E}\left[V_{i}^{\text {one }}\right]\right)+\widehat{U_{i}} R_{i}^{+}-\left(\frac{\kappa S_{i}}{6}-1\right) \\
= & \left(\frac{\kappa S_{i}}{6}-1\right)\left(1-\frac{1}{\widehat{U_{i} R_{i}^{+}}}\right)^{\widehat{U_{i} R_{i}^{+}-1}+\widehat{U_{i}} R_{i}^{+}} \\
& -\left(\frac{\kappa S_{i}}{6}-1\right) .
\end{aligned}
$$

(1) If $\widehat{U}_{i} \leq\lfloor Q / \varsigma\rfloor$, that is, the estimated number of active M2M UE pieces is not higher than the maximum number of participating M2M UE pieces that can be supported by the data channels, in this underload case, the first ACB scheme is not initiated and the first ACB factor is $R_{i}^{+}=1$. Let $S_{i}^{*}$ represent the optimal number of RBs allocated to PRACH, 
and it is achieved when the gap between $C_{i}$ and the number of available PUSCH is minimum.

$$
S_{i}^{*}=\underset{1 \leq S_{i}<Q}{\arg \min }\left\{\left\lfloor\left\lfloor C_{i}\right\rfloor-\left\lfloor\frac{\left(Q-S_{i}\right)}{\varsigma}\right\rfloor\right\},\right.
$$

where $C_{i}$ is given by

$$
C_{i}=\left(\frac{\kappa S_{i}}{6}-1\right)\left(1-\frac{1}{\widehat{U}_{i}}\right)^{\widehat{U_{i}}-1}+\widehat{U}_{i}-\left(\frac{\kappa S_{i}}{6}-1\right)
$$

After $S_{i}^{*}$ is determined, the optimal second ACB factor is obtained by $R_{i}^{*}=\left(\kappa S_{i}^{*} / 6-1\right) / \widehat{U}_{i}$. In this case, $\kappa S_{i}^{*} / 6-$ 1 participating M2M UE pieces select the preambles from $\kappa S_{i}^{*} / 6-1$ nonspecified preambles and $\widehat{U}_{i}-\left(\kappa S_{i}^{*} / 6-1\right)$ participating M2M UE pieces select the specified preamble. The number of successful accesses is expressed as

$$
V_{i}^{\text {succ }}=\min \left\{\left\lfloor\frac{\left(Q-S_{i}\right)}{\varsigma}\right\rfloor,\left\lfloor C_{i}\right\rfloor\right\}_{\text {when } S_{i}=S_{i}^{*}} .
$$

(2) If $\widehat{U}_{i}>\lfloor Q / \varsigma\rfloor$, that is, the estimated number of active M2M UE pieces is higher than the maximum number of participating M2M UE pieces that can be supported by the data channels, in this overload case, the first ACB scheme is initiated and the first ACB factor is $R_{i}^{+}=\lfloor Q / \varsigma\rfloor / \widehat{U}_{i}$, and $\lfloor Q / \varsigma\rfloor$ active M2M UE pieces are allowed to participate in RA procedure. $S_{i}^{*}$ RBs should be allocated to the PRACH such that

$$
S_{i}^{*}=\underset{1 \leq S_{i}<Q}{\arg \min }\left\{\left\lfloor\left\lfloor C_{i}\right\rfloor-\left\lfloor\frac{\left(Q-S_{i}\right)}{\varsigma}\right\rfloor\right\},\right.
$$

where $C_{i}$ is given by

$$
\begin{aligned}
C_{i}= & \left(\frac{\kappa S_{i}}{6}-1\right)\left(\frac{1}{\lfloor Q / \varsigma\rfloor}\right)^{\lfloor Q / \varsigma\rfloor-1}+\left\lfloor\frac{Q}{\varsigma}\right\rfloor \\
& -\left(\frac{\kappa S_{i}}{6}-1\right) .
\end{aligned}
$$

After $S_{i}^{*}$ is determined, the optimal second ACB factor is $R_{i}^{*}=\left(\kappa S_{i}^{*} / 6-1\right) /\lfloor Q / \varsigma\rfloor$. In this case, $\kappa S_{i}^{*} / 6-1$ participating M2M UE pieces select the preambles from $\kappa S_{i}^{*} / 6-1$ nonspecified preambles and $\lfloor Q / \varsigma\rfloor-\left(\kappa S_{i}^{*} / 6-1\right)$ participating M2M UE pieces select the specified preamble. The number of successful accesses is expressed as

$$
V_{i}^{\text {succ }}=\min \left\{\left\lfloor\frac{\left(Q-S_{i}\right)}{\varsigma}\right\rfloor,\left\lfloor C_{i}\right\rfloor\right\}_{\text {when } S_{i}=S_{i}^{*}} .
$$

The joint optimization of the ACB factors and the number of RBs for PRACH for hybrid RA and data transmission is given in Algorithm 1. Therefore, based on the mentioned principle for allocating RBs to PRACH and PUSCH, we assign $S_{i}^{*}$ and $Q-S_{i}^{*}$ RBs from $Q$ available RBs to PRACH and PUSCH, respectively.

\section{Performance Evaluation}

In this section, the performance of the proposed scheme is evaluated in terms of the number of successful accesses in each cycle and the grant time (GT) in comparison with the algorithm in [18, 19]. GT is denoted as the required number of cycles in which all M2M UE pieces successfully obtain their uplink resources granted for data transmission. Consider that the system bandwidth is $20 \mathrm{MHz}$, which is divided into 100 RBs as specified in the standard [12]. Except where noted, we set $\kappa=24$ and $\varsigma=1$. That is, 24 preamble sequences are assigned to the M2M UE pieces in every $6 \mathrm{RBs}$. The number of RBs constituting one PUSCH is 1 . Also, we assume that $50 \mathrm{RBs}$ are reserved for RA and data transmission in each cycle; that is, $Q=50$. There are at least $1 \mathrm{RA}$ slot per $2 \mathrm{LTE}$ frames and at most 10 RA slots per LTE frame; that is, the duration of each RA cycle can be valued from $1 \mathrm{~ms}$ to $20 \mathrm{~ms}$ [29].

Figure 5 shows the comparison of the number of active M2M UE pieces and the expected number of successful accesses under beta distribution activation model. We set $T_{P}=100$ and $N=1000,2000$; that is, 1000,2000 active M2M UE pieces exist in the coverage area of an eNB for data transmission. We can see that the expected number of successful accesses is close to the number of active M2M UE pieces; that is, most of the active M2M UE pieces can successfully transmit data packets, which validates the good performance of the proposed scheme in improving the number of successful accesses.

For a better presentation, we assume that the number of active M2M UE pieces varies from 20 to 100 in each cycle. The followings present the performance of the proposed scheme with a wide range of number of active M2M UE pieces.

In Figure 6, we can see that the actual number of reattempting $\mathrm{M} 2 \mathrm{M}$ UE pieces is very well tracked by the estimated number. Since the total number of active M2M UE pieces include the reattempting M2M UE pieces and the new active M2M UE pieces, then the actual total number of active M2M UE pieces is very well tracked by the estimated total number of active M2M UE pieces. We can see that when $\kappa=24, \varsigma=1$, the number of reattempting M2M UE pieces for $Q=50$ is higher than that for $Q=80$ since more $R B$ s are reserved for $R A$ and data transmission when $Q=80$. We can see that when $Q=80, \kappa=24$, the number of reattempting M2M UE pieces for $\varsigma=3$ is higher than that for $\varsigma=1$ since more RBs are needed for transmitting a data packet when $\varsigma=3$. We can see that when $Q=80, \varsigma=1$, the number of reattempting M2M UE pieces for $\kappa=48$ is close to that for $\kappa=24$. This proves that the value of $\varsigma$ has more effect than the value of $\kappa$ in the proposed scheme.

Figure 7 shows the comparison of the expected number of successful accesses among [18, 19] and the proposed scheme. Using the proposed scheme, the expected number of successful accesses in each cycle is increased compared to $[18,19]$. Furthermore, we observe that the expected number of successful accesses by analysis matches that by simulation. According to [18], the optimal number of participating M2M UE pieces is 40 , which is given by $\kappa Q /(k \varsigma+6)$, while in the proposed scheme the optimal number of participating $\mathrm{M} 2 \mathrm{M}$ 


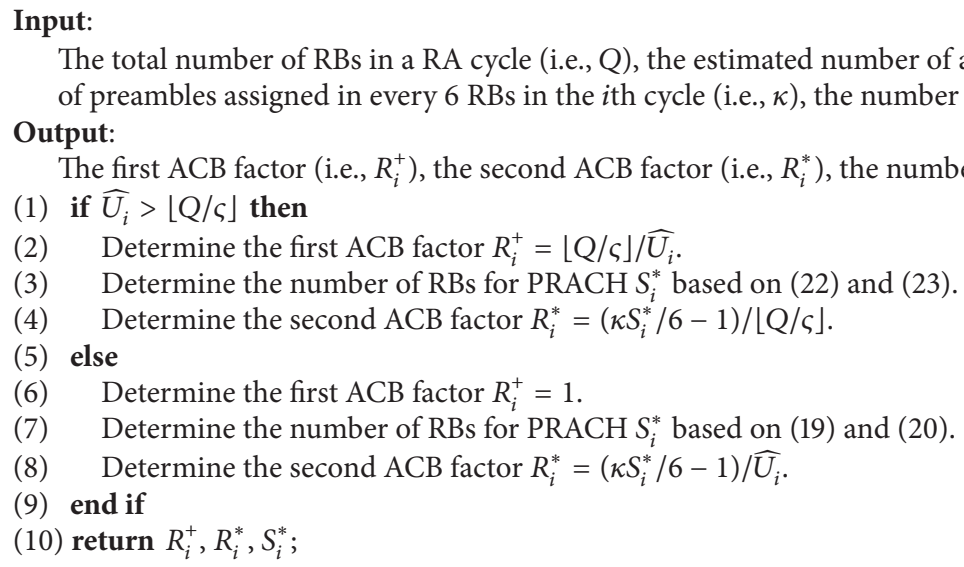

Algorithm 1: Joint optimization of the ACB factors and the number of RBs for PRACH.

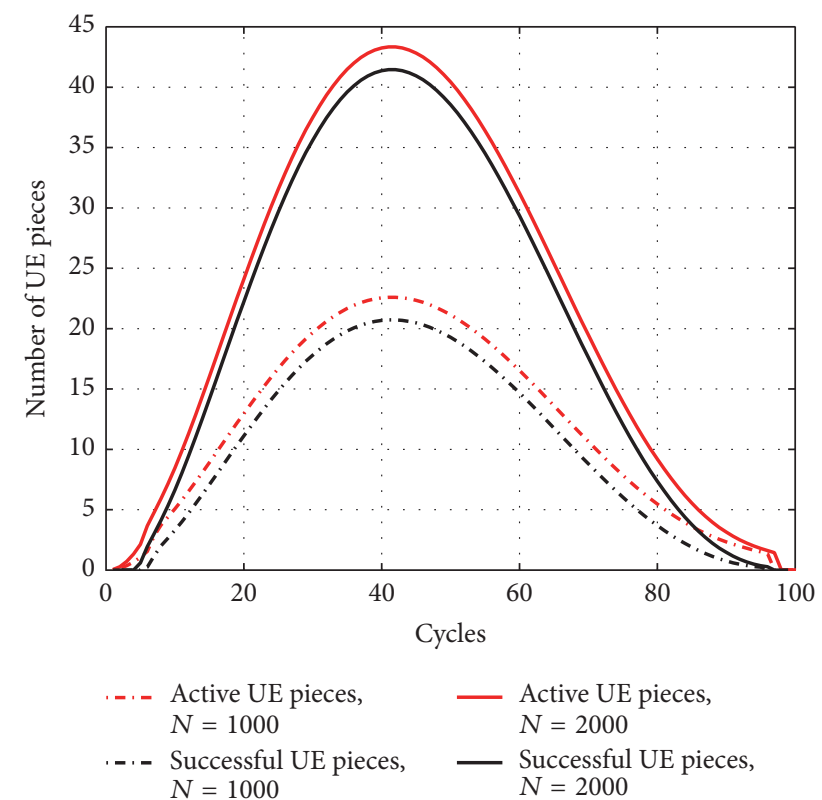

Figure 5: The number of active M2M UE pieces and the expected number of successful accesses for $T_{P}=100$ and $N=1000,2000$ under beta distribution activation model.

UE pieces approximates 50 . The expected number of successful accesses in [19] is higher than that in [18]; this gain comes from the predetection of preamble collisions; therefore, the eNB will not schedule PUSCH to the collided preambles. While it is still less than the number of successful accesses in the proposed scheme, this is because the probability of preamble collisions becomes larger as the number of active M2M UE pieces becomes larger.

Figure 8 shows the GT for different number of M2M UE pieces. As expected, the GT in the proposed scheme is significantly less than that in $[18,19]$. That is because the number of participating M2M UE pieces that successfully obtain their uplink resources granted for data transmission

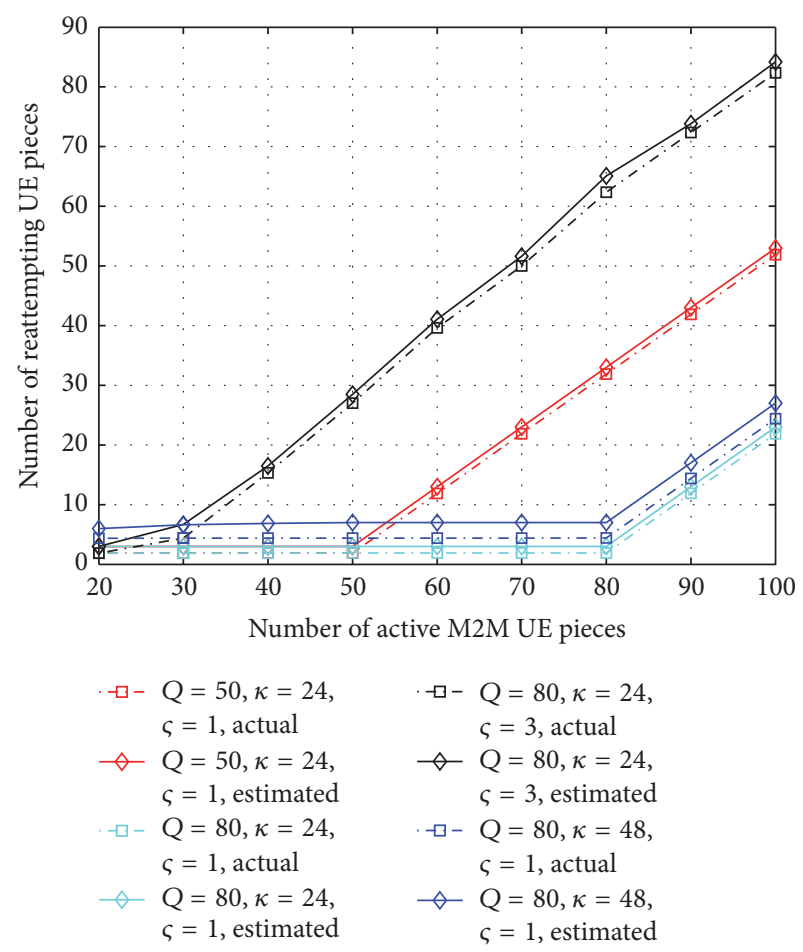

FIGURE 6: The comparison of the expected number of reattempting UE pieces with different $Q, \kappa$, and $\varsigma$.

in the proposed scheme is greater than that in reference schemes.

Figure 9 shows the GT for the total number of RBs reserved for RA and data transmission varying from 25 to 60 . The number of active M2M UE pieces is 60 . As expected, the GT in the proposed scheme is decreased with the increment of the total number of RBs reserved for RA and data transmission; this is because, with the increment of the total number of RBs, the number of participating M2M UE pieces that successfully obtain their uplink resources granted for data transmission increases. 


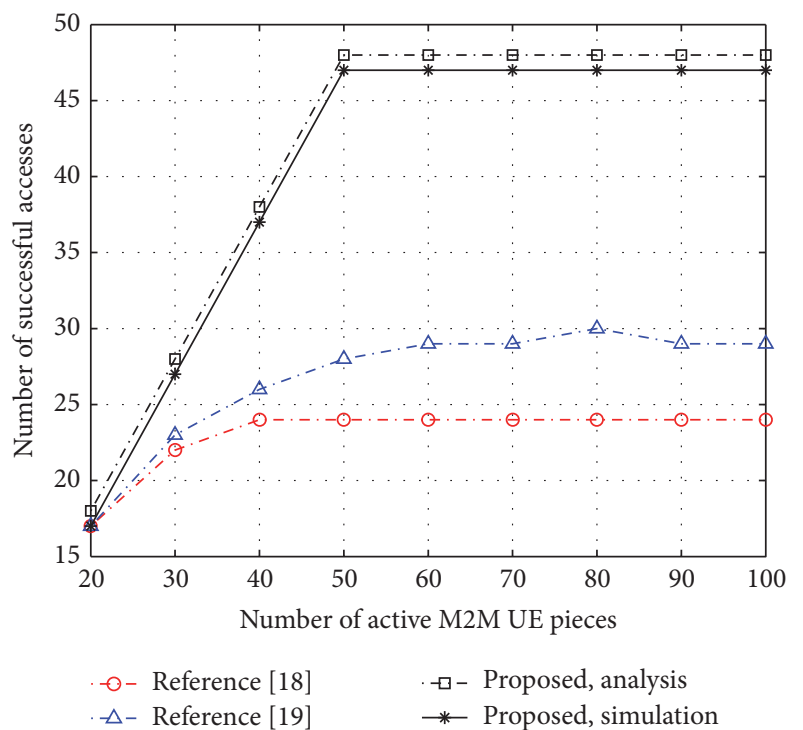

FIGURE 7: The comparison of the expected number of successful accesses among $[18,19]$ and the proposed scheme.

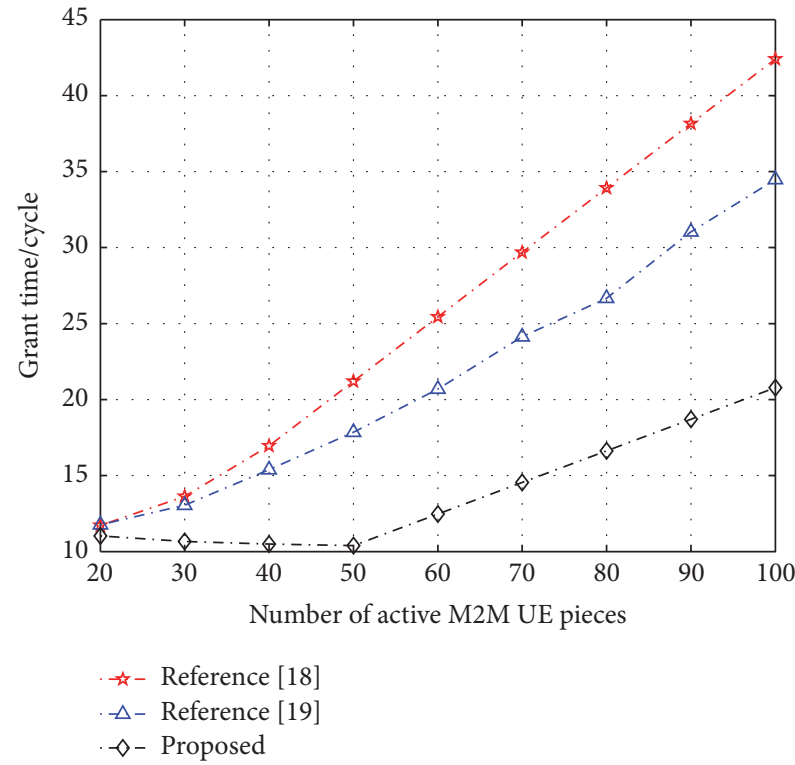

FIgURE 8: The GT versus the number of active M2M UE pieces.

\section{Conclusion}

The paper deals with the RAN congestion problem in the cellular network for M2M communications and proposes a hybrid protocol for RA and data transmission based on twophase ACB mechanisms. A relatively simple load estimation algorithm is designed to estimate the M2M traffic load. In the first phase, the number of participating M2M UE pieces is controlled by the first ACB mechanism according to the estimated traffic load. In the second phase, the participating M2M UE pieces are partitioned into two parts by the second ACB mechanism, which, respectively, select two types of preambles. Uplink resources for RA and data transmission

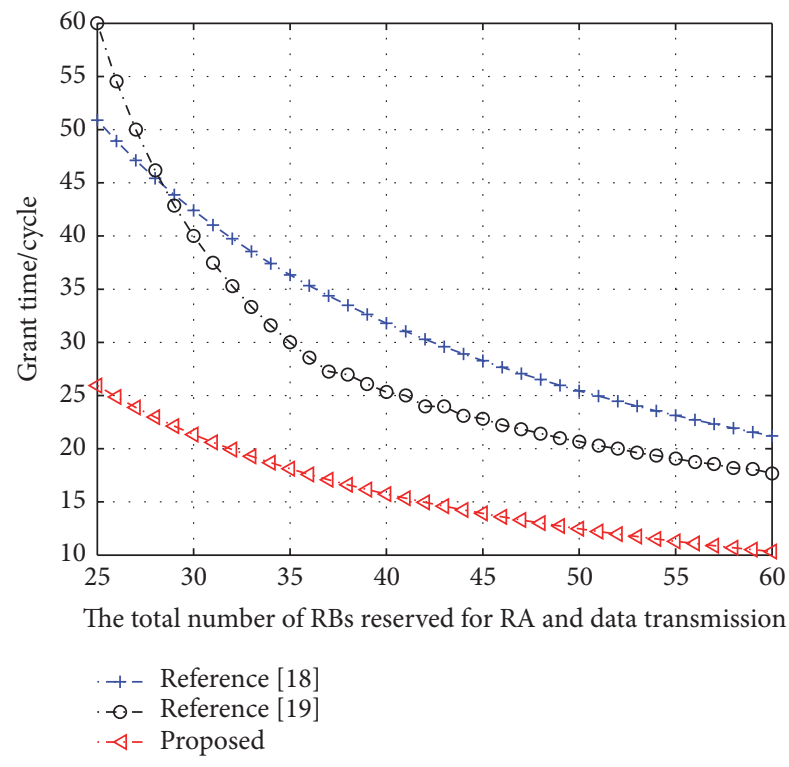

FIGURE 9: The GT versus the number of RBs reserved for RA and data transmission when the number of active M2M UE pieces is 60 .

are granted for the two parts of participating M2M UE pieces to maximize the number of successful accesses. Simulation results show the performance superiority of the proposed scheme in terms of the number of successful accesses and the grant time.

\section{Appendix}

The first ACB factor is $R_{i}^{+}$, and $\widehat{U_{i}} R_{i}^{+}$active M2M UE pieces are allowed to participate in RA procedure. $\operatorname{Pr}\left[q \mid \widehat{U}_{i} R_{i}^{+}\right]$is the probability that, among $\widehat{U}_{i} R_{i}^{+}$participating M2M UE pieces, there are $q \mathrm{M} 2 \mathrm{M}$ UE pieces who pass the second ACB check. $\operatorname{Pr}\left[q \mid \widehat{U_{i}} R_{i}^{+}\right]$is given by

$$
\operatorname{Pr}\left[q \mid \widehat{U_{i}} R_{i}^{+}\right]=\left(\begin{array}{c}
\widehat{U_{i}} R_{i}^{+} \\
q
\end{array}\right) R_{i}^{q}\left(1-R_{i}\right)^{\widehat{U_{i}} R_{i}^{+}-q},
$$

where $R_{i}$ is the second ACB factor. Denote $M_{j \in B_{i}}$ as the number of participating M2M UE pieces who pass the second ACB check and select a nonspecified preamble, named $j$, from $B_{i}$ nonspecified preambles in the $i$ th cycle, where $B_{i}=$ $\kappa S_{i} / 6-1 . \operatorname{Pr}\left[M_{j \in B_{i}}=1 \mid q\right]$ is the probability that only one piece of M2M UE among the $q$ M2M UE pieces selects nonspecified preamble $j, j \in B_{i} \cdot \operatorname{Pr}\left[M_{j \in B_{i}}=1 \mid q\right]$ is given by

$$
\operatorname{Pr}\left[M_{j \in B_{i}}=1 \mid q\right]=\left(\begin{array}{l}
q \\
1
\end{array}\right) \frac{1}{B_{i}}\left(1-\frac{1}{B_{i}}\right)^{q-1}
$$

Denote $V_{M_{j \in B_{i}}=1}$ as the number of successful preambles in the $i$ th cycle, that is, the number of nonspecified preambles that are selected by only one piece of participating $\mathrm{M} 2 \mathrm{M}$ UE. As each preamble is independent of the others, given the event that $q$ participating M2M UE pieces who pass the second $\mathrm{ACB}$ check require access to the eNB, the expected 
number of successful preambles in the $i$ th cycle is given by

$$
\begin{aligned}
\mathrm{E}\left[V_{M_{j \in B_{i}}=1} \mid q\right] & =\sum_{l=1}^{B_{i}} \operatorname{Pr}\left[M_{j \in B_{i}}=1 \mid q\right] \\
& =\sum_{l=1}^{B_{i}}\left(\begin{array}{l}
q \\
1
\end{array}\right) \frac{1}{B_{i}}\left(1-\frac{1}{B_{i}}\right)^{q-1} \\
& =\left(\begin{array}{l}
q \\
1
\end{array}\right)\left(1-\frac{1}{B_{i}}\right)^{q-1} .
\end{aligned}
$$

Therefore, given that the eNB knows $\widehat{U_{i}} R_{i}^{+}$, the expected number of successful preambles is given by

$$
\begin{aligned}
\mathrm{E} & {\left[V_{M_{j \in B_{i}}=1} \mid \widehat{U_{i}} R_{i}^{+}\right] } \\
& =\sum_{q=1}^{\widehat{U_{i}} R_{i}^{+}} \operatorname{Pr}\left[q \mid \widehat{U_{i}} R_{i}^{+}\right] \mathrm{E}\left[V_{M_{j \in B_{i}}=1} \mid q\right] \\
& =\sum_{q=1}^{\widehat{U_{i}} R_{i}^{+}}\left(\begin{array}{c}
\widehat{U_{i}} R_{i}^{+} \\
q
\end{array}\right) R_{i}^{q}\left(1-R_{i}\right)^{\widehat{U_{i}} R_{i}^{+}-q} \\
& \cdot\left(\begin{array}{c}
q \\
1
\end{array}\right)\left(1-\frac{1}{B_{i}}\right)^{q-1}=\widehat{U_{i}} R_{i}^{+} R_{i}\left(1-\frac{R_{i}}{B_{i}}\right)^{\widehat{U_{i} R_{i}^{+}-1}} .
\end{aligned}
$$
obtain

By taking the derivative of (A.4) with respect to $R_{i}$, we

$$
\begin{aligned}
& \frac{d}{d R_{i}} \mathrm{E}\left[V_{M_{j \in B_{i}}=1} \mid \widehat{U_{i}} R_{i}^{+}\right] \\
& \quad=\widehat{U}_{i} R_{i}^{+}\left(1-\frac{R_{i}}{B_{i}}\right)^{\widehat{U_{i}} R_{i}^{+}-2}\left(1-\frac{\widehat{U_{i}} R_{i}^{+} R_{i}}{B_{i}}\right) .
\end{aligned}
$$

When $\widehat{U}_{i} R_{i}^{+} \leq B_{i},\left(d / d R_{i}\right) \mathrm{E}\left[V_{M_{j \in B_{i}}=1} \mid \widehat{U}_{i} R_{i}^{+}\right] \geq 0$, the maximum number of successful preambles is achieved when $R_{i}=1$. When $\widehat{U}_{i} R_{i}^{+}>B_{i}$, let $\left(d / d R_{i}\right) \mathrm{E}\left[V_{M_{j \in B_{i}}=1} \mid \widehat{U_{i}} R_{i}^{+}\right]=0$, and then $R_{i}=B_{i} / \widehat{U_{i}} R_{i}^{+}$. Therefore, we have $R_{i}=\min \left(1, B_{i} /\right.$ $\widehat{U_{i}} R_{i}^{+}$), where $B_{i}=\kappa S_{i} / 6-1$.

\section{Conflicts of Interest}

The authors declare that there are no conflicts of interest regarding the publication of this paper.

\section{Acknowledgments}

This work was supported by the National Natural Science Foundation of China (61501056) and National Science and Technology Major Project of China (no. 2016ZX03001012).

\section{References}

[1] F. Boccardi, R. Heath Jr., A. Lozano, T. L. Marzetta, and P. Popovski, "Five disruptive technology directions for 5G," IEEE Communications Magazine, vol. 52, no. 2, pp. 74-80, 2014.
[2] R. Ratasuk, A. Prasad, Z. Li, A. Ghosh, and M. Uusitalo, "Recent advancements in M2M communications in $4 \mathrm{G}$ networks and evolution towards 5G," in Proceedings of the 18th International Conference on Intelligence in Next Generation Networks (ICIN '15), pp. 52-57, fra, February 2015.

[3] 3GPP TR, "Study on RAN Improvements for machine-type communications," 3GPP TR 3GPP TR 37.868 V11.0.0, 2011.

[4] A. Osseiran, J. F. Monserrat, and Y. P. Marsch, 5G Mobile and Wireless Communications Technology, Cambridge University Press, Cambridge, UK, 2016.

[5] 3GPP TSG, "UE demodulation simulation assumptions," Work Item R4-072182, 3GPP TSG RAN WG4, 2007.

[6] M. Levesque, F. Aurzada, M. Maier, and G. Joos, "Coexistence analysis of $\mathrm{H} 2 \mathrm{H}$ and M2M Traffic in FiWi smart grid communications infrastructures based on multi-tier business models," IEEE Transactions on Communications, vol. 62, no. 11, pp. 39313942, 2014.

[7] 3GPP TS, "Service requirements for Machine Type Communications (MTC)," 3GPP TS 3GPP TS 22.368 Rel 13, 2014.

[8] 3GPP TS, "General Packet Radio Service (GPRS) enhancements for Evolved Universal Terrestrial Radio Access Network (EUTRAN) Access," 3GPP TS 3GPP TS 23.401 Rel 12, 2014.

[9] 3GPP TR, "Study on Machine Type Communications and other mobile data applications communications enhancements," 3GPP TR 3GPP TR 23.887 Rel 12, 2013.

[10] R. Ratasuk, N. Mangalvedhe, Y. Zhang, M. Robert, and J. P. Koskinen, "Overview of narrowband IoT in LTE Rel-13," in Proceedings of the IEEE Conference on Standards for Communications and Networking (CSCN '16), pp. 1-7, Berlin, Germany, October 2016.

[11] 3GPP TR, "Study on extended architecture support for Cellular Internet of Things (CIoT)," 3GPP TR 3GPP TR 23.730 Rel 14, 2016.

[12] 3GPP, "Evolved universal terrestrial radio access (E-UTRA): physical channels and modulation," 3GPP TR 3GPP TR 36.211, V12.2.0, Sophia Antipolis, 2014.

[13] W. Li, Q. Du, L. Liu, P. Ren, Y. Wang, and L. Sun, "Dynamic allocation of RACH resource for clustered M2M communications in LTE networks," in Proceedings of the 4th International Conference on Identification, Information, and Knowledge in the Internet of Things (IIKI '15), pp. 140-145, chn, October 2015.

[14] Y.-C. Pang, G.-Y. Lin, and H.-Y. Wei, "Context-Aware Dynamic Resource Allocation for Cellular M2M Communications," IEEE Internet of Things Journal, vol. 3, no. 3, pp. 318-326, 2016.

[15] M. Tavana, V. Shah-Mansouri, and V. W. S. Wong, "Congestion control for bursty M2M traffic in LTE networks," in Proceedings of the IEEE International Conference on Communications (ICC '15), pp. 5815-5820, London, UK, June 2015.

[16] H. He, Q. Du, H. Song, W. Li, Y. Wang, and P. Ren, "Trafficaware ACB scheme for massive access in machine-to-machine networks," in Proceedings of the IEEE International Conference on Communications (ICC '15), pp. 617-622, London, UK, June 2015.

[17] C.-Y. Oh, D. Hwang, and T.-J. Lee, "Joint access control and resource allocation for concurrent and massive access of M2M devices," IEEE Transactions on Wireless Communications, vol. 14, no. 8, pp. 4182-4192, 2015.

[18] D. T. Wiriaatmadja and K. W. Choi, "Hybrid random access and data transmission protocol for machine-to-machine communications in cellular networks," IEEE Transactions on Wireless Communications, vol. 14, no. 1, pp. 33-46, 2015. 
[19] N. Zhang, G. Kang, J. Wang, Y. Guo, and F. Labeau, "Resource allocation in a new random access for M2M communications," IEEE Communications Letters, vol. 19, no. 5, pp. 843-846, 2015.

[20] A. Zanella, M. Zorzi, A. F. Dos Santos et al., "M2M massive wireless access: challenges, research issues, and ways forward," in Proceedings of the IEEE Globecom Workshops (GC Wkshps '13), pp. 151-156, USA, December 2013.

[21] G. C. Madueno, S. Stefanovic, and P. Popovski, "Efficient LTE access with collision resolution for massive M2M communications," in Proceedings of the IEEE Globecom Workshops (GC Wkshps '14), pp. 1433-1438, USA, December 2014.

[22] A. Laya, L. Alonso, and J. Alonso-Zarate, "Is the random access channel of LTE and LTE-A suitable for M2M communications? A survey of alternatives," IEEE Communications Surveys and Tutorials, vol. 16, no. 1, pp. 4-16, 2014.

[23] N. H. Mahmood, N. Pratas, T. Jacobsen, and P. Mogensen, "On the performance of one stage massive random access protocols in 5G systems," in Proceedings of the IEEE International Symposium on Turbo Codes and Iterative Information Processing, pp. 340-344, Brest, France, September 2016.

[24] 5th Generation Non-orthogonal Waveform for Asynchronous Signalling (5GNOW), "Deliverable D4.2-5GNOW final MAC/networking concepts," Tech. Rep., 2015.

[25] D. Lin, G. Charbit, and I.-K. Fu, "Uplink contention based multiple access for 5G cellular IoT," in Proceedings of the IEEE Vehicular Technology Conference (VTC Fall '15), USA, September 2015.

[26] S. Duan, V. Shah-Mansouri, and V. W. Wong, "Dynamic access class barring for M2M communications in LTE networks," in Proceedings of the IEEE Global Communications Conference (GLOBECOM '13), pp. 4747-4752, Atlanta, GA, USA, December 2013.

[27] ZTE, “ZTE. R2-104662: MTC Simulation Results with Specific Solutions," 3GPP TSG RAN WG2 Meeting ZTE. R2-104662, 2010.

[28] N. Zhang, "An improved random access procedure for M2M communications," in Proceedings of the IEEE Wireless Communications and Networking Conference (WCNC '16), pp. 1-5, Doha, Qatar, April 2016.

[29] S. Sesia, I. Toufik, and M. Baker, LTE-The UMTS Long Term Evolution: Form Theory to Practice, Wiley, 2011. 

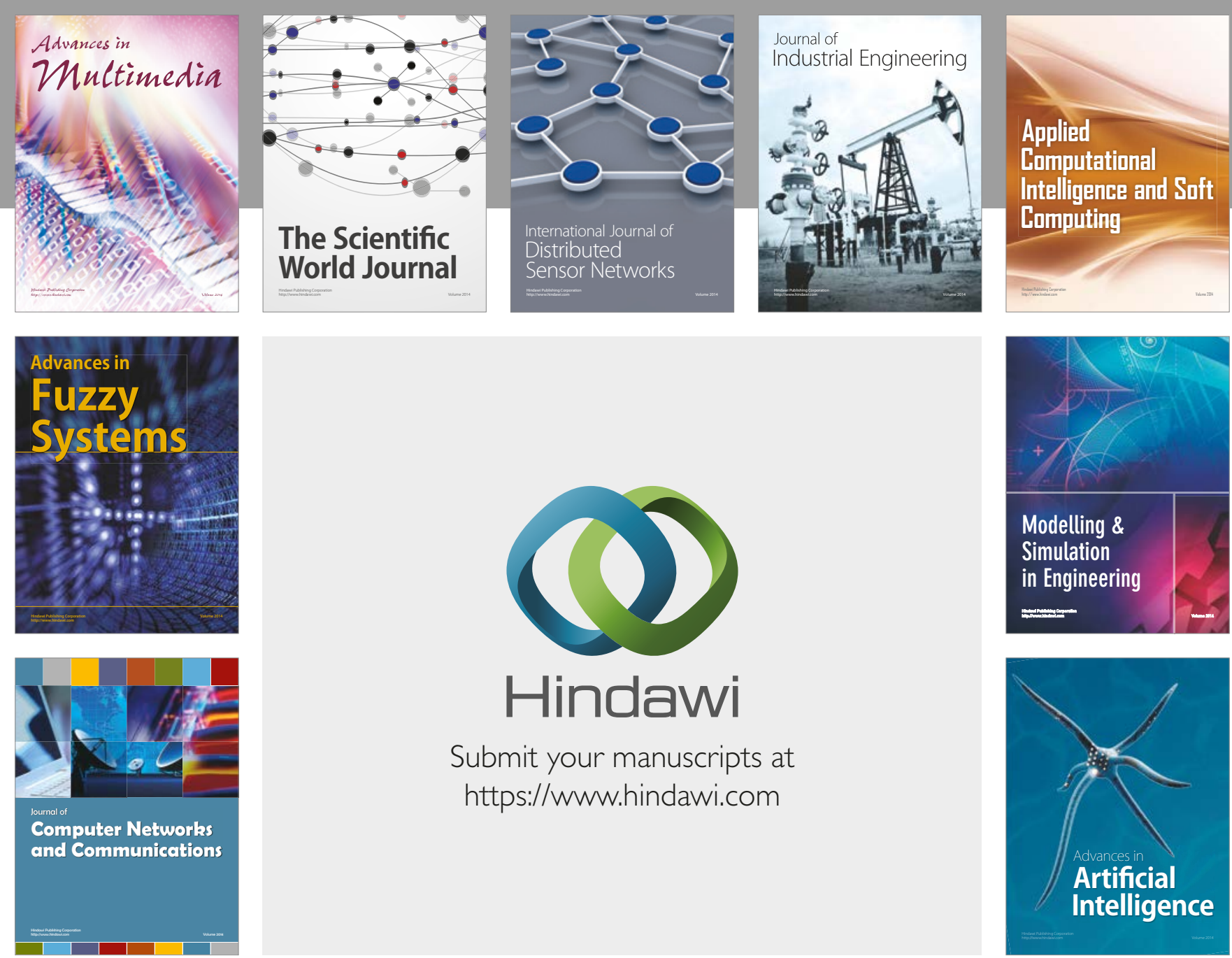

\section{Hindawi}

Submit your manuscripts at

https://www.hindawi.com
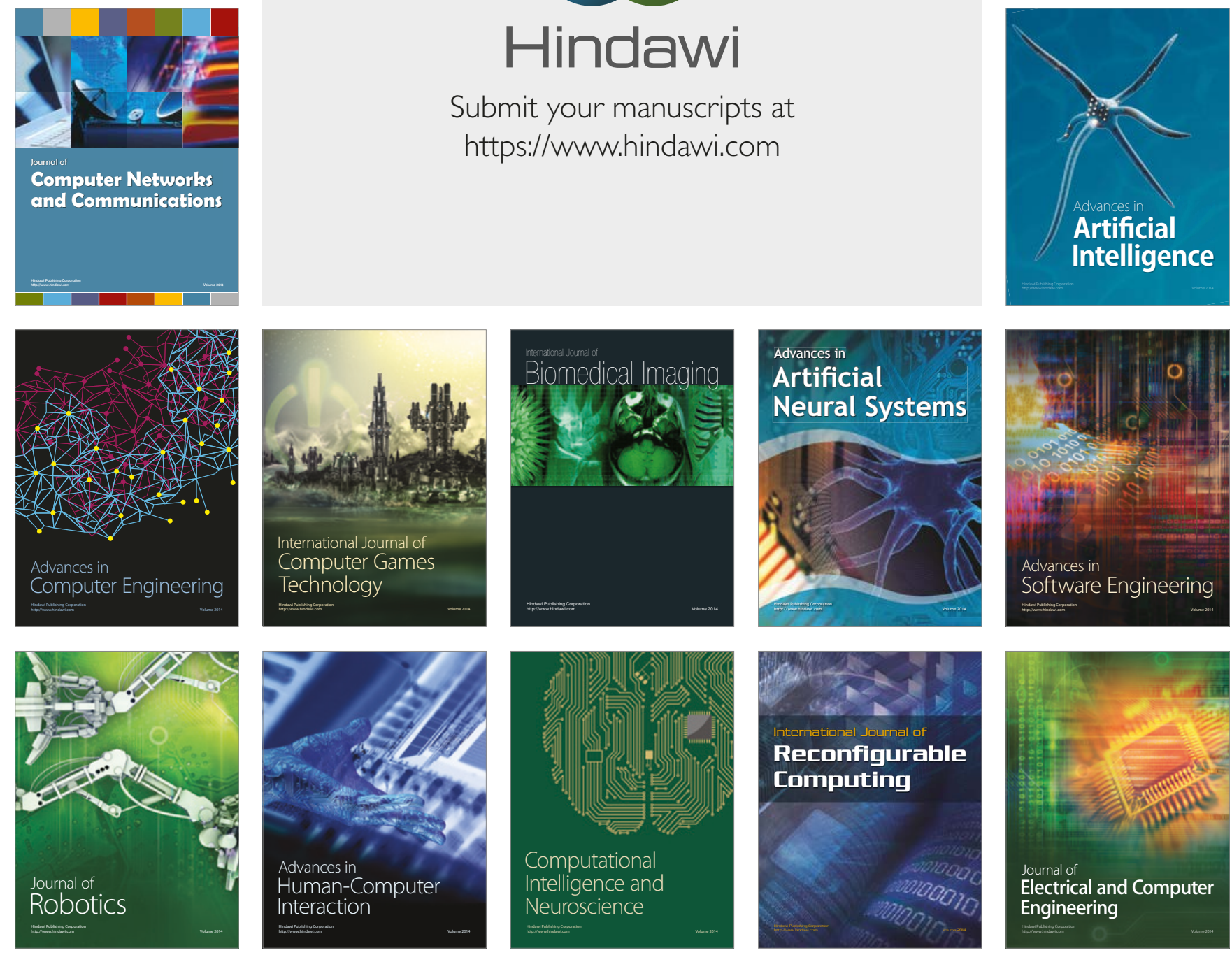